\title{
Inductances Design of High-Frequency Coaxial Transformers
}

\author{
Gierri Waltrich \\ Federal University of Santa Catarina, Joinville, Brazil \\ Email: gierriw@yahoo.com.br
}

Received 15 June 2016; accepted 23 July 2016; published 26 July 2016

Copyright (C) 2016 by author and OALib.

This work is licensed under the Creative Commons Attribution International License (CC BY). http://creativecommons.org/licenses/by/4.0/

(c) () Open Access

\begin{abstract}
In this paper is proposed a methodology to properly determine the leakage and magnetizing inductance of a single-phase high-frequency coaxial transformer. Both, leakage and magnetizing inductance equations are determined considering the transformer dimensions, the ferromagnetic characteristics, and the number of turners. Finite element methods (FEM) and a low scale prototype are also used to validate the equations. The results show that the leakage and magnetizing inductances can be precisely calculated with an error lower than $5 \%$ and $3 \%$, respectively.
\end{abstract}

\section{Keywords}

Inductance, High-Frequency, Coaxial, Transformer

Subject Areas: Electric Engineering

\section{Introduction}

Coaxial transformers are normally used in high-frequency radio applications because it confines the flux leakage inside of the transformer windings [1]. However, it is also possible to find some applications on the power electronics field [2] [3]. For instance, in [4] is shown a successfully implementation of a high-frequency coaxial transformers in a power level of $120 \mathrm{~kW}$. In [5], it is demonstrated that in soft-switching and resonant converters, coaxial transformers might reduce the transformer leakage inductances, and, consequently, decrease the converter commutation losses. Therefore, a methodology to determine, both, magnetizing and leakage inductances of coaxial transformers is developed in this paper, mainly for power electronics applications.

\section{Design of a Two Winding Coaxial Transformer}

In this section, the design of the leakage and the magnetizing inductances of a coaxial transformer with two windings are described. 
To calculate the leakage inductance, a coaxial transformer described in [3] is used. For convenience, this model is repeated here in Figure 1. This transformer is composed by a cooper tube, representing the primary winding, and a Litz cable, which represents the transformer secondary winding. To simplify the method description, initially, the secondary side will be calculated considering only one winding, and later, it might be substituted by $n$ turns.

Leakage inductances can be determined in different ways. In this paper, the inductances are obtained calculating first the magnetic field and energy in different parts of the transformer, and subsequently, obtaining the total leakage inductance. Thus, to carry out the calculations, the coaxial transformer can be divided in four zones, as shown in Figure 2. To calculate only the leakage inductance, imagine primary and secondary sides with the same current density, however, with opposite directions of the currents. For this situation, the magnetic field in zone four is zero, similarly as in a coaxial cable. Obtained the magnetic field in the four zones, the energy can be determined in each zone by

$$
W=\frac{1}{2} \int_{V} \boldsymbol{B} \cdot \boldsymbol{H} \mathrm{d} V=\frac{1}{2} L I^{2}[J]
$$

Thus, applying this method for each zone, and summing the four resulting energies, the leakage inductance (per meter) in a coaxial transformer can be determined by

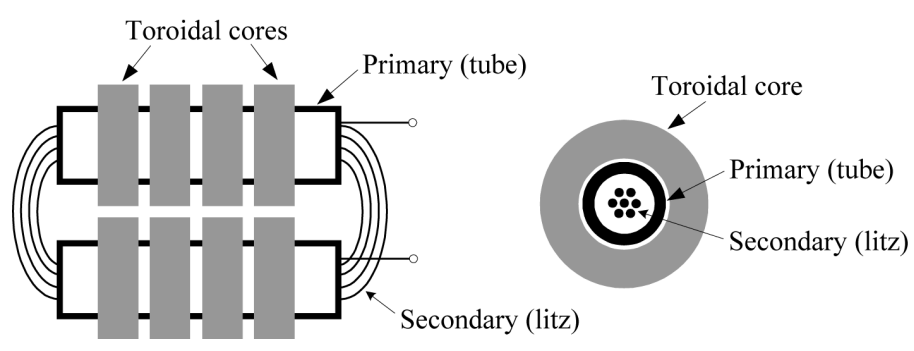

Figure 1. Two-winding coaxial transformer.

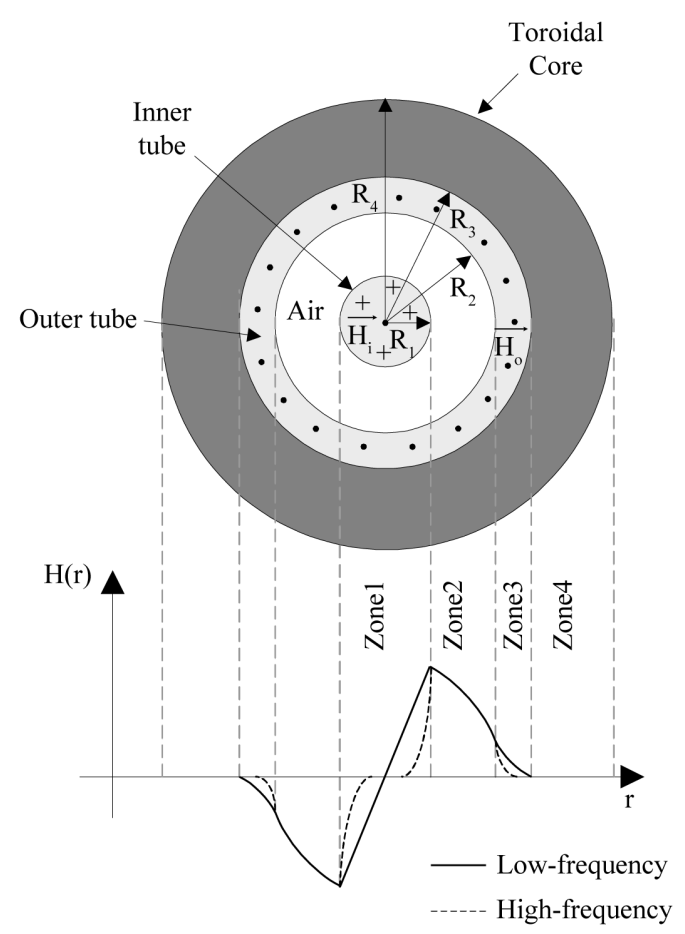

Figure 2. Magnetic field profile of a coaxial transformer cross section area, considering direct current circulating in the primary winding and this same current returning from the secondary winding. 


$$
\frac{L_{l k}}{m}=\frac{\mu_{0} N^{2}}{2 \pi}\left[\frac{1}{4}+\ln \left(\frac{R_{2}}{R_{1}}\right)+\frac{R_{3}^{4}}{\left(R_{3}^{2}-R_{2}^{2}\right)^{2}} \ln \left(\frac{R_{3}}{R_{2}}\right)+\frac{\left(R_{2}^{2}-3 R_{3}^{2}\right)}{4\left(R_{3}^{2}-R_{2}^{2}\right)}\right]\left[\frac{H}{m}\right],
$$

where the symbol $\mu_{o}$ is the permeability of free space, $N$ is the transformer turn ratio, and $R_{1}, R_{2}$, and $R_{3}$ are shown in Figure 2.

To validate the theoretical calculation obtained in (2), Finite Element Methods (FEM) were developed using Maxwell software and the results are shown in Figure 3. By this figure, it is proved that when both primary and secondary windings have the same density current but with opposite direction, the energy stays concentrated in zones one, two, and three, and the energy in zone four in zero. Thus, with Maxwell software is possible to obtain the total energy in those three areas, and, consequently, calculate the leakage inductance and compare with (2).

A 2D FEM numerical model of the coaxial transformer in Figure 2 was developed using the Maxwell software, to calculate the energy in the four zones of the transformer, and the results are shown in Figure 3 . When a current with the same value in the outer tube and inner tube is imposed with opposite direction, the energy will be concentrated in zones 1 to 3, as shown in Figure 3, which represents the leakage inductance. The numerical calculations confirm that the result found in (2) is correct. The methodology, so far, was described for low frequency current. However, when higher-frequencies currents are used, skin effect might be relevant, and it should be considered. For this new situation, the current will be concentrated closer to the cable (or tube) superficies of the primary and the secondary sides, and, therefore, the magnetic field profile will change, as illustrated in Figure 2. Thus, the leakage inductance must be recalculated subtracting the skin-depth, from radii $R_{1}$ and $R_{3}$.

The next step is to calculate the coaxial transformer magnetizing inductance. By applying the same methodology described to obtain the leakage inductance, the magnetizing inductance can be theoretically determined by

$$
\frac{L_{m g}}{m}=\frac{\mu_{o} \mu_{f e} N^{2}}{2 \pi} \ln \left(\frac{R_{4}}{R_{3}}\right)\left[\frac{H}{m}\right],
$$

where the symbol $\mu_{f e}$ represents the relative permeability of the ferrite. Equation (3) is obtained considering the current passing through one of the transformer sides, i.e., primary or secondary side. The total energy this time is concentrated in the core (zone four), as shown in Figure 3, and just a very low amount of energy stays in zones 1, 2 and 3, and therefore, this energy is disregarding.

Energy $\left[\mathrm{J} / \mathrm{m}^{3}\right]$
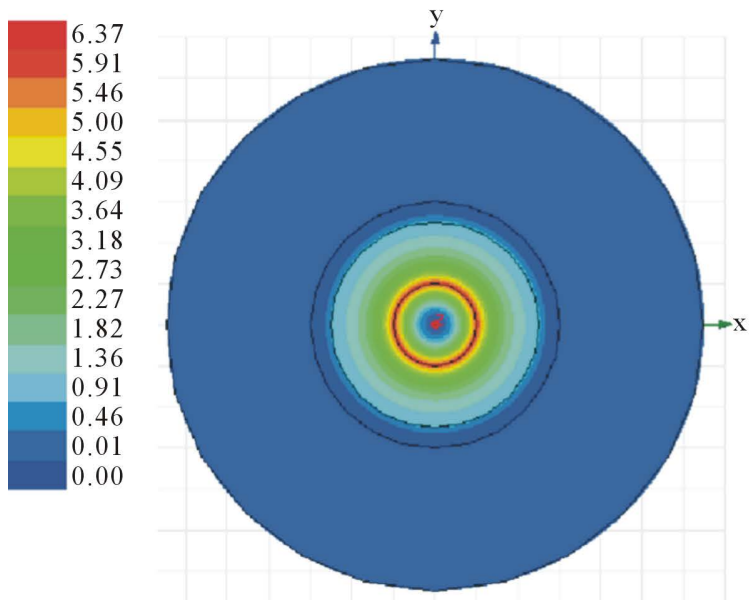

(a)
Energy $\left[\mathrm{J} / \mathrm{m}^{3}\right]$
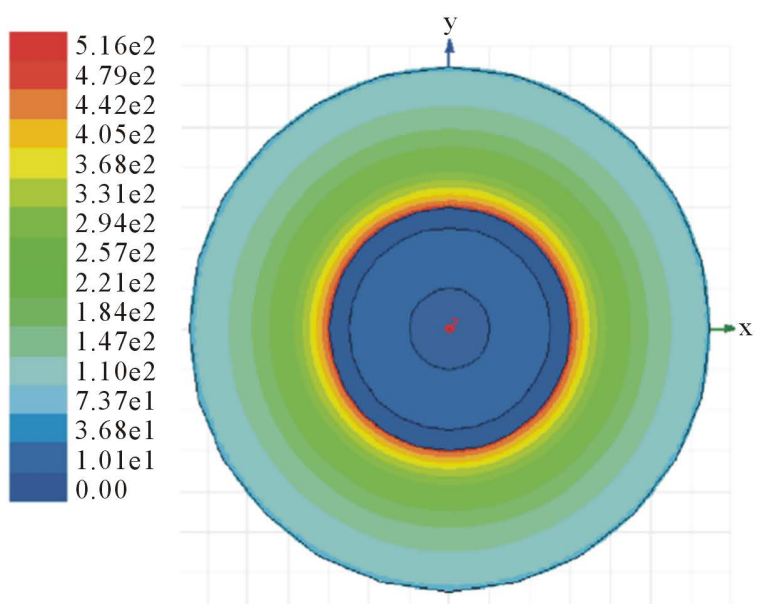

(b)

Figure 3. Energy within the four zones of Figure 2, obtained with a 2D Maxwell model when the density current for both, primary and sencondary windings, are equal, but with opposite direction (a), and when there is current in only one transformer side (b). 
With the leakage and the magnetizing inductances, calculated by (2) and (3), respectively, a two by two matrix inductance for this coaxial transformer can be obtained, and it can be compared with the matrix inductance generated by the Maxwell software, to validate the theoretical models.

Using Maxwell software it is also possible to generate a 3D model to evaluate the fringing flux effect in the external cables in a real application as shown in Figure 4.

\section{Inductance Matrix for a Three-Winding Transformer}

For a three-winding transformer, the inductance matrix is defined as

$$
\left[\begin{array}{l}
u_{1} \\
u_{2} \\
u_{3}
\end{array}\right]=\left[\begin{array}{ccc}
L_{1} & M_{12} & M_{13} \\
M_{21} & L_{2} & M_{23} \\
M_{31} & M_{32} & L_{3}
\end{array}\right] \frac{\mathrm{d}}{\mathrm{d} t}\left[\begin{array}{c}
i_{1} \\
i_{2} \\
i_{3}
\end{array}\right] .
$$

Figure 5 shows the circuit diagram for the $\pi$-model using three-windings. Because the inductance matrix and circuit diagram both have six parameters, this system is uniquely determined. The six parameters from Figure 5 are found to be

$$
\begin{array}{ll}
L_{L 1}=L_{1}-\frac{M_{12} M_{13}}{M_{23}}, & M=\frac{M_{12} M_{13}}{M_{23}}, \\
L_{L 2}=L_{2}-\frac{M_{12} M_{23}}{M_{13}}, & N_{2}=\frac{M_{23}}{M_{13}}, \\
L_{L 3}=L_{3}-\frac{M_{13} M_{23}}{M_{12}}, & N_{3}=\frac{M_{23}}{M_{12}} .
\end{array}
$$

To calculate these six parameters in a real transformer, six steps must be carried out: measure three reflected magnetizing inductances on each side, considering the other two windings open, then short-circuit two windings and measure the remaining two leakage inductances for all windings. For transformers with more than three windings a $\pi$-model is over determined.

\section{Experimental Results}

To validate the theoretical and the finite element results, prototypes of the coaxial transformer, shown in Figure 1, with one turn for both primary and secondary windings, were built. Two prototypes were manufactured: one with a cooper tube of $28.6 \mathrm{~cm}$ long and another one with two cooper tubes, where each tube is $14.3 \mathrm{~cm}$ long. The picture of both prototypes is shown in Figure 7. The relative permeability $\left(\mu_{f e}\right)$ of the cores used in the experimentations are equal to 700 .

To determine the matrix inductance the T-model, described in [6], is used. This model is shown in Figure 6 and the inductance matrix given by this model is

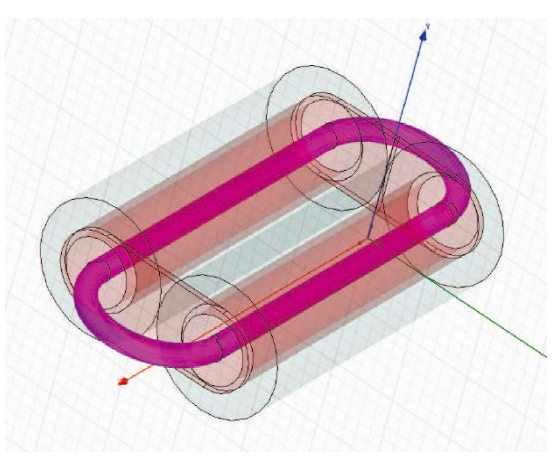

(a)

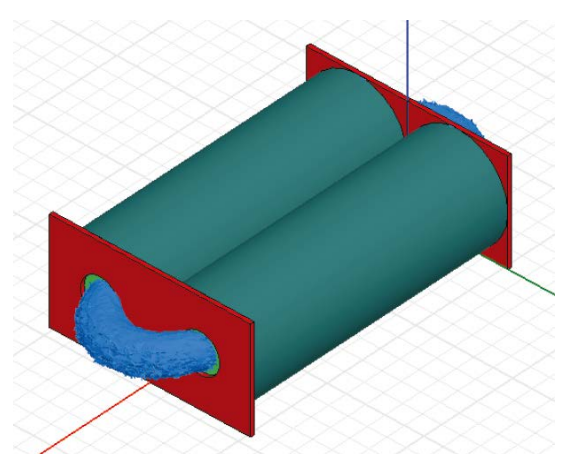

(b)

Figure 4. Energy obtained with a 3D Maxwell model. 


$$
\left[\begin{array}{l}
u_{1} \\
u_{2}
\end{array}\right]=\left[\begin{array}{ll}
L_{1} & M \\
M & L_{2}
\end{array}\right] \frac{\mathrm{d}}{\mathrm{d} t}\left[\begin{array}{l}
i_{1} \\
i_{2}
\end{array}\right] .
$$

The equation given by (6) is represented by the circuit shown in Figure 6 . In this figure we assume $M_{12}=M_{21}=$ $M$. The primary and secondary leakage inductance equations are defined based on Figure 6, in accordance with

$$
L_{l p}=L_{1}-N M \text { and } L_{l s}=L_{2}-\frac{M}{N} .
$$

Table 1 summarizes the results obtained from experimental measurements using an impedance analyzer. A 3D FEM model was developed only for the two-tube model because most of the leakage inductance in the one-tube prototype is concentrated inside of the core. Thus, the fringing flux of the prototype from Figure 7 was neglected.

Theoretical, simulated, and experimental results were obtained at $10 \mathrm{kHz}$. Theory and simulation considered the skin effect.
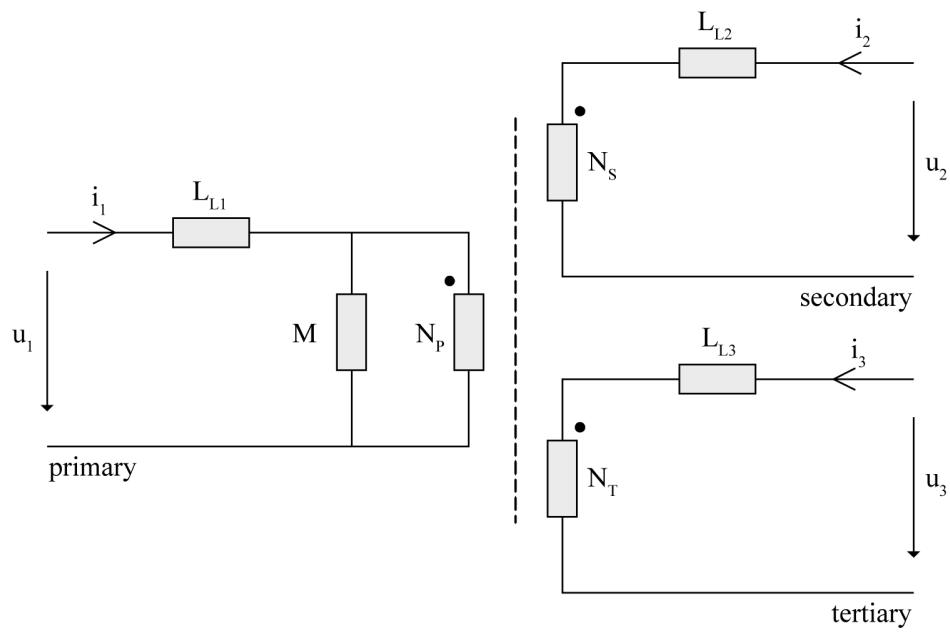

Figure 5. Circuit diagram of $\pi$-model for a three-winding transformer.

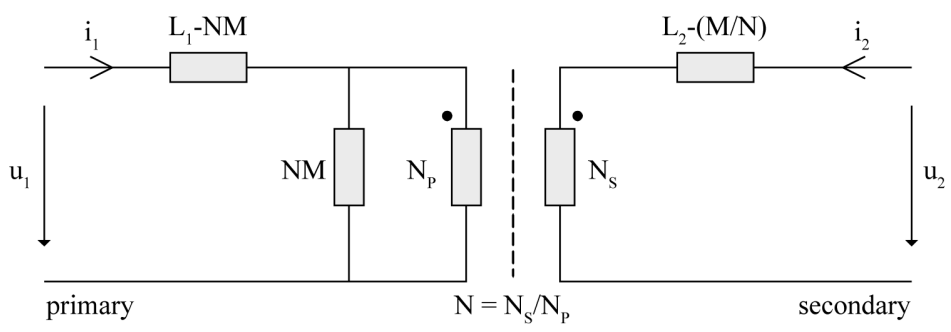

Figure 6. T-model circuit for a two-winding transformer.

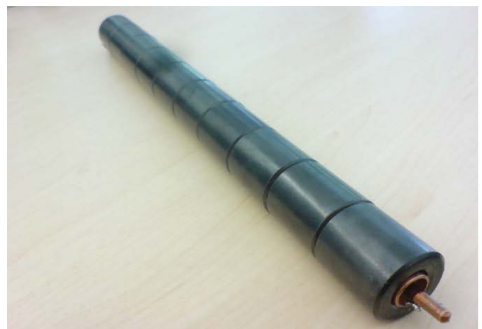

(a)

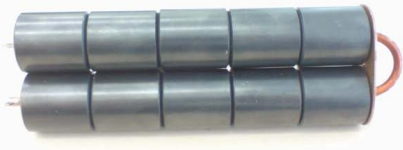

(b)

Figure 7. Coaxial transformer prototype built for one (a) and two tubes (b). 
Table 1. Results for the coaxial transformer designed.

\begin{tabular}{ccc}
\hline & Leakage $[\mathrm{nH}]$ & Magnetizing $[\mu \mathrm{H}]$ \\
\hline Theoretical (1 tube) & 65.13 & 28.22 \\
Theoretical (2 tubes) & 65.13 & 28.22 \\
3D Maxell software (2 tubes) & 96.61 & 28.34 \\
Experimental (1 tube) & 68.54 & 27.75 \\
Experimental (2 tubes) & 92.99 & 27.61 \\
\hline
\end{tabular}

The theoretical, the simulated, and the experimental inductance matrix of the configuration described in Figure 7, are found to be

$$
\begin{aligned}
& \text { Theoretical }(\mu \mathrm{H}) \quad \text { Simulation }(\mu \mathrm{H}) \quad \text { Experimental }(\mu \mathrm{H}) \\
& {\left[\begin{array}{ll}
28.22 & 28.23 \\
28.23 & 28.30
\end{array}\right]\left[\begin{array}{ll}
28.22 & 28.22 \\
28.22 & 28.29
\end{array}\right] \quad\left[\begin{array}{ll}
27.46 & 27.57 \\
27.57 & 27.75
\end{array}\right] \text {. }}
\end{aligned}
$$

\section{Conclusions}

A methodology to determine the inductances of a coaxial transformer was developed in this paper. If the dimensions and material characteristics used to build the transformer are known, the leakage and magnetizing inductances can be precisely calculated. All results were obtained in three ways: theoretically, by Finite Element Methods, and with measurements on an experimental setup.

The magnetizing inductance of this transformer is quite low $(\sim 28 \mu \mathrm{H})$. To obtain a higher magnetizing inductance, three changes can be made: increasing the number of turns of the outer tube, increasing the toroidal core dimensions, or increasing both dimensions and number of turns.

\section{References}

[1] Mason, W.P. and Sykes, R.A. (1937) The Use of Coaxial and Balanced Transmission Lines in Filters and Wide-Band Transformers for High Radio Frequencies. Bell System Technical Journal, 16, 275-302. http://dx.doi.org/10.1002/j.1538-7305.1937.tb00422.x

[2] Waltrich, G. (2013) Energy Management of Fast-Charger Systems for Electric Vehicles-Experimental. Ph.D. Dissertation, TU/e, Eindhoven.

[3] Waltrich, G., Duarte, J.L. and Hendrix, M.A.M. (2010) Multiport Converters for Fast Chargers of Electrical Vehicles-Focus on High-Frequency Coaxial Transformers. Power Electronics Conference (IPEC), 2010 International, Sapporo, 21-24 June 2010, 3151-3157. http://dx.doi.org/10.1109/IPEC.2010.5543381

[4] Klontz, K.W., Divan, D.M. and Novotny, D.W. (1995) An Actively Cooled 120 kW Coaxial Winding Transformer for Fast Charging Electric Vehicles. IEEE Transactions on Industry Applications, 31, 1257-1263. http://dx.doi.org/10.1109/28.475695

[5] Kheraluwala, M.N., Gascoigne, R.W., Divan, D.M. and Baumann, E.D. (1992) Performance Characterization of a High-Power Dual Active Bridge DC-to-DC Converter. IEEE Transactions on Industry Applications, 28, 1294-1301. http://dx.doi.org/10.1109/28.175280

[6] Hayes, J.G., O’Donovan, N. and Egan, M.G. (2004) The Extended T Model of the Multiwinding Transformer. Power Electronics Specialists Conference, 2004. PESC 04. 2004 IEEE 35th Annual, 3, 1812-1817. 
Submit or recommend next manuscript to OALib Journal and we will provide best service for you:

- Publication frequency: Monthly

- 9 subject areas of science, technology and medicine

- Fair and rigorous peer-review system

- Fast publication process

- Article promotion in various social networking sites (LinkedIn, Facebook, Twitter, etc.)

- Maximum dissemination of your research work

Submit Your Paper Online: Click Here to Submit

Contact Us: service@oalib.com 